\title{
Clinical presentations, management outcomes, and diagnostic dilemma in Kocuria endophthalmitis
}

\author{
Vivek Pravin Dave ${ }^{1 *}$, Joveeta Joseph ${ }^{2}$, Avinash Pathengay $^{3}$ and Rajeev R. Pappuru ${ }^{1}$
}

\begin{abstract}
Aim: To describe the clinical presentations and management outcomes of Kocuria endophthalmitis and discuss diagnostic dilemmas

Design: Retrospective interventional comparative case series

Intervention: Eight unilateral cases with culture-proven Kocuria endophthalmitis from January 2013 to December 2017 underwent vitrectomy/vitreous biopsy and intravitreal antibiotic with or without additional procedures. The undiluted vitreous was subjected to microbiologic evaluation.

Main outcome measures: The mean age at presentation, etiology, number of interventions, interval between inciting event and presentation, type of intravitreal antibiotic used, and anatomic and functional outcomes were reported. A favorable anatomic outcome was defined as preservation of the globe, absence of hypotony, attached retina, and absence of active inflammation at the last visit.
\end{abstract}

Results: In the current series, there were five males and three females. The mean age at presentation was $31 \pm 17$. 44 years (median 30 years). The inciting event was open globe injury in five cases and one case each following cataract surgery, microbial keratitis, and endogenous cause. Visual acuity was $\geq 20 / 400$ in one case at presentation and $\geq 20 / 400$ in three cases at the last visit. The species identified by Vitek 2, included Kocuria kristinae in three, $K$. rosea in four and $K$. varians in one. Follow-up period was $8.25 \pm 8.24$ months (median 6.5). Six cases (75\%) had complete resolution of infection and inflammation at the last visit. Anatomic success was achieved in $75 \%$.

Conclusions: Kocuria is a relatively rare cause of endophthalmitis often misdiagnosed as Staphylococcal endophthalmitis. Clinical presentation can be variable but favorable antibiotic susceptibility and appropriate timely management can result in acceptable visual and anatomic outcomes.

Keywords: Endophthalmitis, Kocuria, Coagulase-negative Staphylococci

\section{Summary statement}

Kocuria species can mimic other coagulase-negative Staphylococci and cause diagnostic dilemma. Rarely, Kocuria can be a cause of endophthalmitis. In the current communication, we describe our experience of diagnosing and managing endophthalmitis due to Kocuria spp.

\footnotetext{
* Correspondence: vivekoperates@yahoo.co.in

'Smt. Kanuri Santhamma Center for Vitreoretinal Diseases, Kallam Anji Reddy Campus, LV Prasad Eye Institute, Hyderabad, Telangana 500034, India Full list of author information is available at the end of the article
}

\section{Introduction}

Kocuria are gram-positive coccoid bacteria of the family Micrococcaceae (order Actinomycetales, class Actinobacteria). They are found as tetrads or irregular clusters and are biochemically catalase-positive and coagulase-negative. Kocuria infections over the years have been reported largely as systemic infections in immunocompromised hosts $[1,2]$. Though Kocuria has been known to be a part of the normal microbiota, it can cause infections like peritonitis, endocarditis, cholecystitis, pneumonitis, and urinary catheter-related infections [3, 4-16]. Kocuria infection prevalence could be falsely low as phenotypic assays used for organism identification often misdiagnose Kocuria as Staphylococci [17]. In the 
current communication, we report the presentations, management outcomes, and diagnostic dilemma in a series of Kocuria endophthalmitis treated at our center.

\section{Methods}

This was a retrospective consecutive interventional case series. This was a retrospective chart review of cases with culture-positive Kocuria endophthalmitis. We included all cases with culture-positive Kocuria endophthalmitis from January 2013 to March 2018 at our tertiary eye care center. The study protocol was approved by the institutional review board. The medical records of the patients were retrieved from the medical records department and the microbiology laboratory database. The collected demographic information included the etiology of the infection, interval from the inciting event to the start of symptoms, interval between the start of symptoms and treatment intervention, comorbid systemic illnesses, visual acuity at presentation, detailed biomicroscopic examination including information on media clarity on indirect ophthalmoscopy, retinal examination, ultrasound findings, treatment provided, and final functional and anatomic outcomes.

\section{Management protocol}

Management of the patients depended on their clinical presentation. Cases with milder presentations or with poor corneal clarity underwent vitreous biopsy. Those with severe vitritis as confirmed clinically or on B scan underwent pars plana vitrectomy wherever the corneal clarity allowed the same. All patients were initially empirically treated with intravitreal vancomycin $(1 \mathrm{mg} / 0.1$ $\mathrm{ml})$ and ceftazidime $(2.25 \mathrm{mg} / 0.1 \mathrm{ml})$ after collecting the vitreous sample. The vitreous sample was collected from the mid-vitreous cavity with a 2 -cc syringe plugged into the suction tubing of the vitreous cutter on cutting mode and before opening the infusion fluid. Further injections were based on the culture report. If no response was noted, a repeat intravitreal injection or a pars plana vitrectomy was done after $48-72 \mathrm{~h}$ depending on the corneal clarity. In some patients, additionally, anterior chamber fluid and the explanted intraocular lens were also processed. Vitreous samples were transported to the microbiology laboratory immediately and examined by direct microscopy (Calcofluor white, Gram, Giemsa stains) and cultured on appropriate bacterial (aerobic and anaerobic) and fungal media. All media were incubated at $37^{\circ} \mathrm{C}$ for 1 week except SDA which was incubated at $25^{\circ} \mathrm{C}$. Only significant culture results were considered. Growth on two or more media or confluent growth on at least one solid medium at the site of inoculation or growth on one medium with consistent direct microscopy result was defined as a significant positive culture. The culture isolates were further identified by biochemical tests and Vitek 2 (Biomerieux, France). The organisms were identified using the Vitek 2 ID GPC gram-positive identification card which uses a fluorogenic methodology for organism identification. After the initial surgical intervention, the patients received topical antibiotics like ciprofloxacin $0.3 \%$, cycloplegics, and topical steroids. Systemically, the patients were administered oral ciprofloxacin $750 \mathrm{mg}$ twice a day empirically.

\section{Outcome definition}

The outcome at the last visit was evaluated in terms of anatomic and functional outcome. A favorable anatomic outcome was defined as preservation of the globe, absence of hypotony, attached retina, and absence of active inflammation at the last visit. A favorable functional outcome was defined as an attached retina with a vision of $\geq 20 / 400$ at the last visit.

\section{Results}

The current case series included eight eyes of eight patients. There were five males and three females. The mean age at presentation was $31 \pm 17.44$ years (median 30 years). None of the patients had any systemic illness and all were immunocompetent. The inciting event was open globe injury in five cases and cataract surgery, microbial keratitis, and endogenous cause in one case each. The case with microbial keratitis had a corneal ulcer perforation and at presentation already had a primary procedure of a tissue adhesive application done elsewhere.

The ulcer finally resolved, and at the last visit, the patient showed a vascularized corneal scar. Of the three patients with post-trauma endophthalmitis who presented with a corneal tear, all show a favorable anatomic outcome while two also had a favorable visual outcome. One case presenting with endogenous endophthalmitis developed a quiet anterior chamber but had persistent vitritis at the last visit. The eye with an open globe injury with scleral involvement developed a recurrent retinal detachment with an overall poor anatomic and functional outcome. The clinical features, the type of intervention, the number of injections, and the medications used are summarized in Table 1. The presenting visual acuity was favorable in one case, while at the final visit, it was favorable in three cases. At the last visit, endophthalmitis was noted to be resolved in six cases. The tissue sample that tested positive for Kocuria sp. varied with the cases and is summarized in Table 2.

The direct microscopy of the vitreous fluid showed the presence of gram-positive cocci in pairs, groups, and chains (Figs. 1 and 2) under gram stain in four of eight cases in our series. Presence of Kocuria sp. was confirmed by growth of large, cream-colored colonies on chocolate and/or blood agar (Fig. 3) from the vitreous fluid in seven of eight cases and from AC Tap in one of 


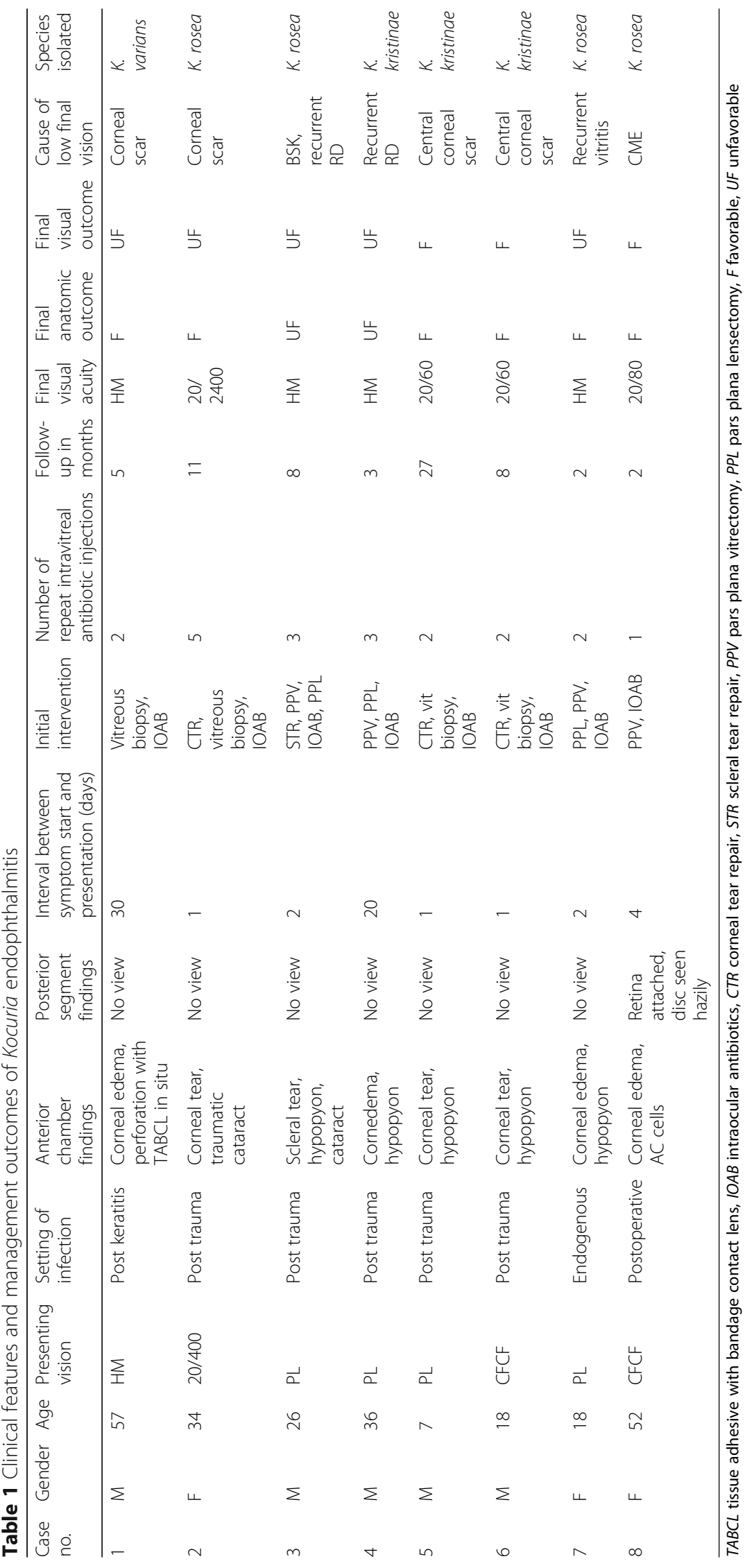


Table 2 Smear and culture positivity for various tissue samples in the current series

\begin{tabular}{lllll}
\hline Case & AC smear & AC culture & Vitreous smear & Vitreous culture \\
\hline 1 & - & + & - & - \\
2 & - & - & + & + \\
3 & - & - & - & + \\
4 & - & - & - & + \\
5 & - & - & - & + \\
6 & - & $+*$ & + & + \\
7 & + & - & + & $+{ }^{\#}$ \\
8 & - & - & + & + \\
\hline
\end{tabular}

$A C$ anterior chamber

*AC culture-positive for Aspergillus niger not for Kocuria

\#Culture-positive for Kocuria and also for Streptococcus species

eight cases. All of these isolates were catalase positive and coagulase negative. Vitek 2 identification revealed the presence of Kocuria rosea in four cases, K. kristinae in three cases, and $K$. varians in one case using the GP card. Overall antibiotic susceptibility to most commonly used antibiotics was found to be good (Table 3). Figures 4, 5, 6, and 7 depict clinical features at presentation and final visits of a few cases.

\section{Discussion}

The current report describes the outcome of a series of cases of Kocuria endophthalmitis. In a retrospective study from China describing the causative organisms in infectious endophthalmitis, 1/330 cases reported was reported as Kocuria [17]. While cases of Kocuria keratitis have been reported previously with variable visual outcome $[18,19]$, this is the first detailed description of endophthalmitis caused by Kocuria sp. Kocuria is a member of the Micrococcaceae family and currently includes 18 species $[1,2,20]$. Though it is ubiquitous in

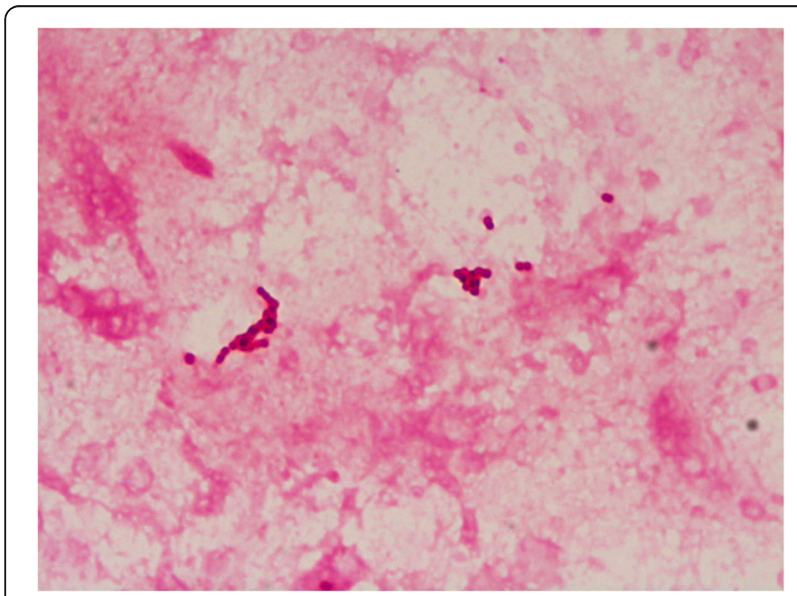

Fig. 1 Gram stain $(\times 100)$ of vitreous sample (case 2$)$ showing grampositive cocci in pair and short-chain 0-6/oil immersion field

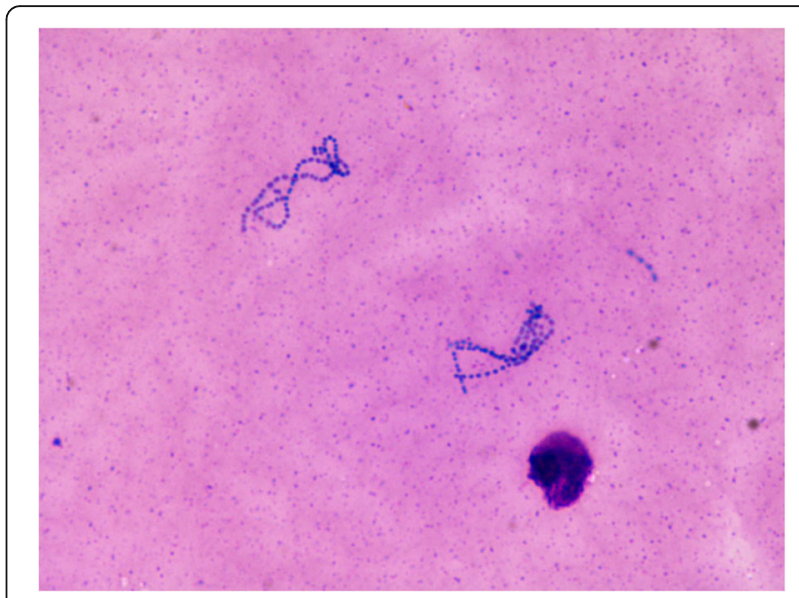

Fig. 2 Giemsa stain $(\times 100)$ of vitreous sample (case 7) showing gram-positive cocci in chains 0-plenty/oil immersion field

nature and freely found as normal skin and oral flora in humans, only 5/18 known species are known to be opportunistic pathogens [1]. The diagnostic difficulties in Kocuria identification lie in the fact that the phenotypic assays used for microbe identification often misdiagnose Kocuria as coagulase-negative Staphylococci [21]. Further diagnostic difficulty occurs due to a differential behavior of Kocuria in vitro and in vivo especially in stress conditions $[22,23]$. Pathological strains are known to be more phenotypically variable then saprophytic strains [24-27]. Identification of Kocuria spp. remains elusive because most clinical microbiology laboratories have limited or no access to advanced molecular techniques. Laboratory identification of Kocuria spp. can be made conventionally only on a high index of suspicion. Properties such as morphological variability between these

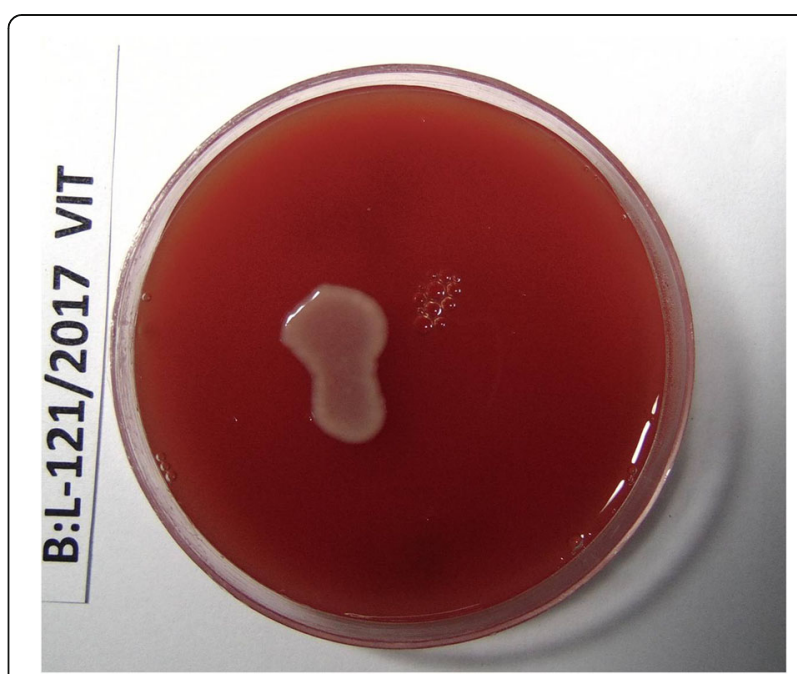

Fig. 3 Culture of vitreous sample (case 2) showing large smooth convex cream-colored colonies on blood agar after 48-h incubation which was identified on Vitek 2 as Kocuria rosea 
Table 3 Case-wise isolated Kocuria species and antibiotic susceptibility

\begin{tabular}{|c|c|c|c|c|c|c|c|c|}
\hline Case number & Species & Amikacin & Cefazolin & Chloramphenicol & Ciprofloxacin & Moxifloxacin & Vancomycin & Cefuroxime \\
\hline 1 & K. varians & + & + & + & + & + & + & + \\
\hline 2 & K. rosea & + & + & + & + & + & + & + \\
\hline 3 & K. rosea & + & + & + & + & + & + & + \\
\hline 4 & K. kristinae & - & - & + & + & + & + & + \\
\hline 5 & K. kristinae & + & + & + & + & + & + & + \\
\hline 6 & K. kristinae & + & + & + & + & + & + & + \\
\hline 7 & K. rosea & - & + & + & + & + & + & + \\
\hline 8 & K. rosea & + & + & + & + & + & + & + \\
\hline
\end{tabular}

bacteria and other similar gram-positive cocci, as well as biochemical properties including the antimicrobial susceptibility patterns against selective antibiotics, could be used to presumptively identify Kocuria spp. Susceptibility towards bacitracin and lysozyme and resistance to nitrofurantoin, furazolidone, and lysostaphin can be used to separate this bacterium from Staphylococci. This however is not commonly done in routine work-ups. Increase in time-dependent pigmentation of the colonies can also lead to a diagnosis of Kocuria as the typical pigmentation of these colonies increase with time especially after a culture period of $48 \mathrm{~h}$ [1]. The introduction of the Vitek 2 GP-card database allows for a better identification of Kocuria in the recent times [28]. Though there are reports of coagulase-negative Staphylococci being wrongly identified as Kocuria by the Vitek 2 system [21], other more recent studies indicate a good reliability of the Vitek 2 system $[29,30]$. Our case series includes cases that have been identified by Vitek 2 system alone. The clinical features of the cases in this series included findings like open globe trauma, hypopyon, vitritis, repeat intravitreal antibiotic injections, and an overall fair resolution of the vitritis. These clinical features largely mimic those of endophthalmitis following a Staphylococcus endophthalmitis.

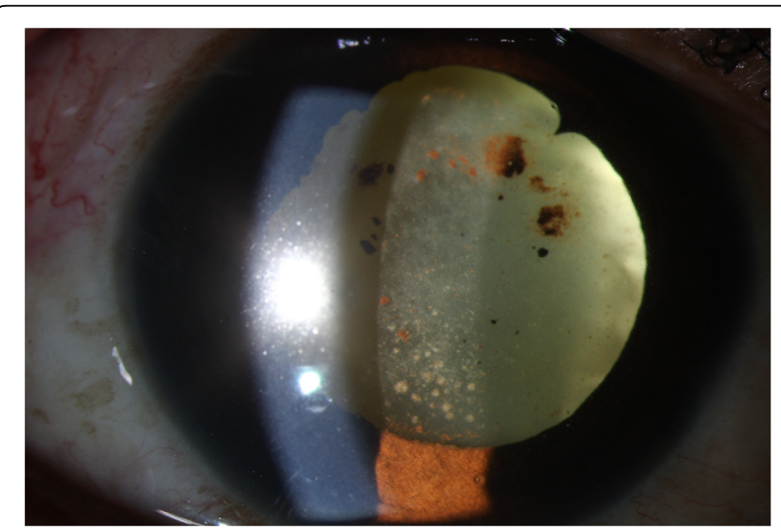

Fig. 4 Preoperative slit lamp photograph of endogenous Kocuria endophthalmitis case
For testing antibiotic susceptibilities, proper guidelines do not exist for Kocuria. Hence, susceptibility breakpoints for Staphylococci are used instead. This can cause a misdiagnosis of either the sensitivity or the resistance pattern in these organisms. Savini et al. [1], in their report, discussed the implications of this misdiagnosis which can lead to recurrent infections and a morbid clinical outcome. It has also been shown that most pathogenic species are susceptible to broad-spectrum antibiotics. In the current reported series, similarly, six of eight cases of Kocuria endophthalmitis reported resolution of infection and inflammation at the last visit. Among the three that did not, two had recurrent retinal detachment and one had corneal decompensation which possibly added to the persistence of the inflammation. This relatively favorable outcome could be attributed to a favorable antibiotic susceptibility pattern as seen in Table 3.

The current study has a few inherent limitations. The effect of various confounding factors could not be independently assessed due to the retrospective nature of the study. The limited sample size did not allow us to assess any parameters that could have influenced the outcome. A proportion of cases of endophthalmitis in this series were post trauma. Trauma itself is a confounding factor for a final unfavorable visual outcome. Thus, it would be

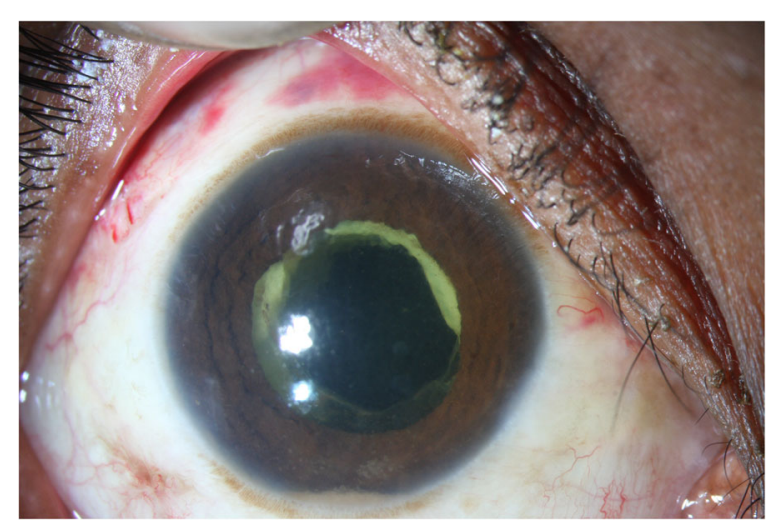

Fig. 5 Postoperative slit lamp photograph of the endogenous case following pars plana vitrectomy and lensectomy 


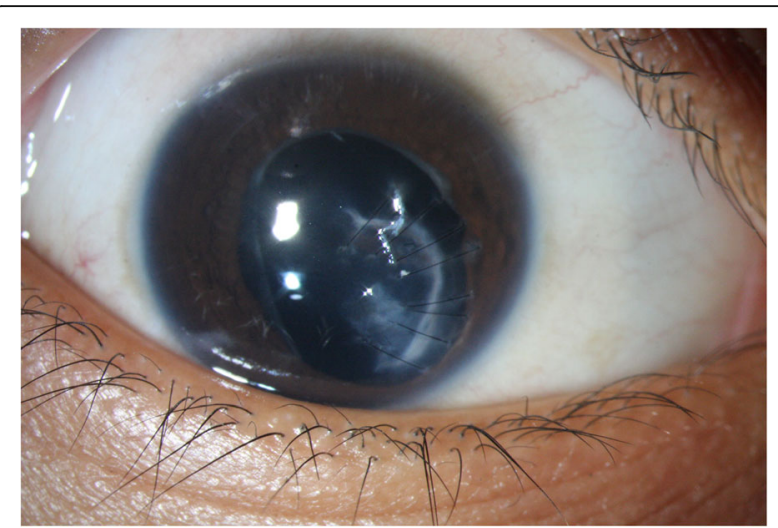

Fig. 6 Postoperative photograph of resolved Kocuria endophthalmitis following open globe trauma with a repaired corneal laceration

difficult to clearly delineate in the post-traumatic subset, whether the unfavorable visual outcome is due to trauma or due to the subsequent endophthalmitis. Though $75 \%$ cases in the current study had resolution of endophthalmitis at the last follow-up, visual improvement is limited due to anterior segment opacities. Further corneal intervention for those patients is pending as this manuscript is being written. Following corneal intervention, further visual improvement can be expected. In conclusion, Kocuria endophthalmitis is a relatively rare but emerging cause of endophthalmitis and is often misdiagnosed as CoNS endophthalmitis. The occurrence of Kocuria spp. in patients with endophthalmitis should not be ignored as contaminants. Though clinical presentation can often be variably late, timely and appropriate management with multiple interventions as and when required can result in an acceptable visual and anatomic outcome.

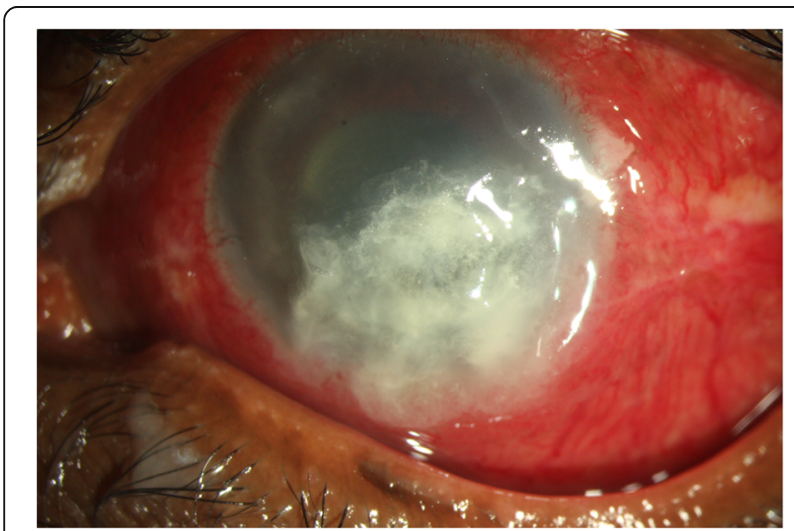

Fig. 7 Preoperative slit lamp photograph of a case of Kocuria endophthalmitis post perforated corneal ulcer with tissue adhesive in situ

\section{Acknowledgements}

Not applicable

\section{Funding}

Funding was received from the Hyderabad Eye Research foundation to analyze the microbiology samples.

Availability of data and materials

Please contact the author for data requests.

\section{Authors' contributions}

VPD carried out the manuscript writing and proofreading and drafted the final copy. JJ carried out the microbiologic assessment. AP and RRP participated in its design and coordination and helped to draft the manuscript. All authors read and approved the final manuscript.

\section{Ethics approval and consent to participate}

Ethics committee approval was taken from the LV Prasad Eye Institute Hyderabad Ethics committee and Institutional Review board for this research work. No animals were used in this research : Not applicable

\section{Consent for publication}

Please include a Consent for publication - Not applicable as no identity revealing photographs or data of any patient were used in the manuscript : Not Applicable

\section{Competing interests}

The authors declare that they have no competing interests.

\section{Publisher's Note}

Springer Nature remains neutral with regard to jurisdictional claims in published maps and institutional affiliations.

\section{Author details}

'Smt. Kanuri Santhamma Center for Vitreoretinal Diseases, Kallam Anji Reddy Campus, LV Prasad Eye Institute, Hyderabad, Telangana 500034, India.

${ }^{2} J$ haveri Microbiology Center, Brien Holden Eye Research Center, LV Prasad Eye Institute, Hyderabad, India. ${ }^{3}$ Retina and Uveitis Department, GMR Varalaxmi Campus, LV Prasad Eye Institute, Hanumanthawaka Chowk, Visakhapatnam, Andhra Pradesh 530040, India.

Received: 22 August 2018 Accepted: 6 November 2018

Published online: 20 November 2018

\section{References}

1. Savini V, Catavitello C, Masciarelli G et al (2010) Drug sensitivity and clinical impact of members of the genus Kocuria. J Med Microbiol 59:1395-1402

2. Stackebrandt E, Koch C, Gvozdiak O, Schumann P (1995) Taxonomic dissection of the genus Micrococcus: Kocuriagen. nov.,Nesterenkoniagen. nov.,Kytococcusgen. nov., Dermacoccusgen. nov., and Micrococcus Cohn 1872 gen. emend. Int J Syst Bacteriol 45:682-692

3. Basaglia G, Carretto E, Barbarini D et al (2002) Catheter-related bacteremia due to Kocuria kristinae in a patient with ovarian cancer. J Clin Microbiol 40:311-313

4. Altuntas F, Yildiz O, Eser B, Gundogan K, Sumerkan B, Cetin M (2004) Catheter-related bacteremia due to Kocuria rosea in a patient undergoing peripheral blood stem cell transplantation. BMC Infect Dis 4:62

5. Ma ESK, Wong CL, Lai KT, Chan EC, Yam WC, Chan AC (2005) Kocuria kristinae infection associated with acute cholecystitis. BMC Infect Dis 5:60

6. Becker K, Rutsch F, Vekötter A et al (2008) Kocuria rhizophila adds to the emerging spectrum of micrococcal species involved in human infections. J Clin Microbiol 46:3537-3539

7. Lee JY, Kim SH, Jeong HS et al (2009) Two cases of peritonitis caused by Kocuria marina in patients undergoing continuous ambulatory peritoneal dialysis. J Clin Microbiol 47:3376-3378

8. Lai CC, Wang JY, Lin SH et al (2011) Catheter-related bacteraemia and infective endocarditis caused by Kocuria species. Clin Microbiol Infect 17:190-192

9. Tsai CY, Su SH, Cheng YH, Chou YL, Tsai TH, Lieu AS (2010) Kocuria varians infection associated with brain abscess: a case report. BMC Infect Dis 10:102 
10. Carlini A, Mattei R, Lucarotti I, Bartelloni A, Rosati A (2011) Kocuria kristinae: an unusual cause of acute peritoneal dialysis-related infection. Perit Dial Int 31:105-107

11. Cheung CY, Cheng NH, Chau KF, Li CS (2011) An unusual organism for CAPD-related peritonitis: Kocuria kristinae. Perit Dial Int 31:107-108

12. Dunn R, Bares S, David MZ (2011) Central venous catheter-related bacteremia caused by Kocuria kristinae: case report and review of the literature. Ann Clin Microbiol Antimicrob 10:31

13. Moissenet D, Becker K, Mérens A, Ferroni A, Dubern B, Vu-Thien H (2012) Persistent bloodstream infection with Kocuria rhizophila related to a damaged central catheter. J Clin Microbiol 50:1495-1498

14. Meletis G, Gogou V, Palamouti M et al (2012) Catheter-related relapsing peritonitis due to Kocuria varians in a patient undergoing continuous ambulatory peritoneal dialysis. Nefrologia 32:541-542

15. Karadag Oncel E, Boyraz MS, Kara A (2012) Black tonque associated with Kocuria (Micrococcus) kristinae bacteremia in a 4-month-old infant. Eur J Pediatr 171:593

16. Citro R, Prota C, Greco L et al (2013) Kocuria kristinae endocarditis related to diabetic foot infection. J Med Microbiol 62:932-934

17. Duan F, Wu K, Liao J, Zheng Y, Yuan Z, Tan J, Lin X (2016) Causative microorganisms of infectious endophthalmitis: a 5-year retrospective study. J Ophthalmol 2016:6764192

18. Pedro-Aguilar L, Ramirez-Miranda A, Bautista-de Lucio VM, Navas A, OrtizCasas M, Gruae-Hernandez EO (2016) Epidemiology and outcomes of Kocuria keratitis. Eye Contact Lens 42:e20-e24

19. Inada N, Shoji J, Yamagami S (2017) Atopic keratoconjunctivitis complicated by Kocuria koreensis keratitis: the first case. Allergy Asthma Clin Immunol 13:6

20. Stackebrandt E, Frederiksen W, Garrity GM et al (2002) Report of the ad hoc committee for the re-evaluation of the species definition in bacteriology. Int J Syst Evol Microbiol 52:1043-1047

21. Ben-Ami R, Navon-Venezia S, Schwartz D, Carmeli Y (2003) Infection of a ventriculoatrial shunt with phenotypically variable Staphylococcus epidermidis masquerading as polymicrobial bacteremia due to various coagulase-negative Staphylococci and Kocuria varians. J Clin Microbiol 41: 2444-2447

22. Ben-Ami R, Navon-Venezia S, Schwartz D, Schlezinger Y, Mekuzas Y, Carmeli Y (2005) Erroneous reporting of coagulase-negative Staphylococci as Kocuria spp. by the Vitek 2 system. J Clin Microbiol 43:1448-1450

23. Savini V, Catavitello C, Bianco A, Balbinot A, D'Antonio D (2009) Epidemiology, pathogenicity and emerging resistances in Staphylococcus pasteuri: from mammals and lampreys, to man. Recent Pat Anti Infect Drug Discov 4:123-129

24. Christensen GD, Baddour LM, Madison BM et al (1990) Colonial morphology of Staphylococci on Memphis agar: phase variation of slime production, resistance to b-lactam antibiotics, and virulence. J Infect Dis 161:1153-1169

25. Deighton M, Pearson S, Capstick J, Spelman D, Borland R. Phenotypic variation of

26. Ziebuhr W, Heilmann C, Gotz F et al (1997) Detection of the intercellular adhesion gene cluster (ica) and phase variation in Staphylococcus epidermidis blood culture strains and mucosal isolates. Infect Immun 65: 890-896

27. Szczerba I (2003) Susceptibility to antibiotics of bacteria from genera Micrococcus, Kocuria, Nesternkonia, Kytococcus and Dermacoccus. Med Dosw Mikrobiol 55:75-80 Polish

28. Funke G, Funke-Kissling P (2005) Performance of the new Vitek 2 GP card for identification of medically relevant gram-positive cocci in a routine clinical laboratory. J Clin Microbiol 43:84-88

29. Boudewijns M, Vandeven J, Verhaegen J, Ben-Ami R, Carmeli Y (2005) Vitek 2 automated identification system and Kocuria kristinae. J Clin Microbiol 43:5832

30. Purty S, Saranathan R, Prashanth K, Narayanan K, Asir J, Devi CS et al (2013) The expanding spectrum of human infections caused by Kocuria species: a case report and review of literature. Emerg Microbes Infect 2:71

\section{Submit your manuscript to a SpringerOpen ${ }^{\circ}$ journal and benefit from:}

- Convenient online submission

- Rigorous peer review

- Open access: articles freely available online

- High visibility within the field

- Retaining the copyright to your article

Submit your next manuscript at $\boldsymbol{\nabla}$ springeropen.com 